\title{
Nifuratel, a novel STAT3 inhibitor with potent activity against human gastric cancer cells
}

This article was published in the following Dove Press journal:

Cancer Management and Research

I November 2017

Number of times this article has been viewed

\author{
Hailun Zheng', 2,* \\ Huang Hong ${ }^{1, *}$ \\ Lulu Zhang ${ }^{1,2, *}$ \\ Xiong Cai ${ }^{1,2}$ \\ Meng $\mathrm{Hu}^{1,2}$ \\ Yuepiao $\mathrm{Cai}^{2}$ \\ Bin Zhou' \\ Jiayuh $\operatorname{Lin}^{3}$ \\ Chengguang Zhao ${ }^{2}$ \\ Wanle $\mathrm{Hu}^{\prime}$ \\ 'Department of Coloproctology, \\ The Second Affiliated Hospital \\ and Yuying Children's Hospital \\ of Wenzhou Medical University, \\ ${ }^{2}$ Chemical Biology Research Center, \\ School of Pharmaceutical Sciences, \\ Wenzhou Medical University, \\ Wenzhou, Zhejiang, People's \\ Republic of China; ${ }^{3}$ Department of \\ Biochemistry and Molecular Biology, \\ University of Maryland Greenebaum \\ Comprehensive Cancer Center, \\ University of Maryland School of \\ Medicine, Baltimore, MA, USA \\ *These authors contributed equally to \\ this work
}

Correspondence: Chengguang Zhao Chemical Biology Research Center, School of Pharmaceutical Sciences,

Wenzhou Medical University, Building

II, Chashan Street, University Town,

Wenzhou 325035, Zhejiang, People's

Republic of China

Tel/fax +865778669 9057

Email zhaocheng.uang@।63.com

Wanle Hu

The Second Affiliated Hospital and Yuying Children's Hospital of Wenzhou Medical University, 109 Xueyuanxi Road, Lucheng

District, Wenzhou 32500, Zhengjiang,

People's Republic of China

Tel/fax +865778800 2609

Email hw19993@126.com

\begin{abstract}
Activation of the signal transducer and activator of transcription 3 (STAT3) is observed in multiple cancer types, including gastric cancer, and represents a potential drug target for chemotherapy. Currently, clinically available small-molecule inhibitors targeting STAT3 are lacking. Here, we report that nifuratel, an antiprotozoal and antifungal drug, is a potent inhibitor of STAT3. We found that nifuratel significantly suppressed proliferation and induced apoptosis of gastric cancer cells. Studies of the mechanism of action of nifuratel indicated that it acts by inhibiting the constitutive and interleukin-6-induced STAT3 activation. Taken together, our findings demonstrate that nifuratel may be a novel, clinically accessible STAT3 inhibitor in gastric cancer cells.
\end{abstract}

Keywords: nifuratel, STAT3, gastric cancer, inhibitor, drug

\section{Introduction}

Gastric cancer is one of the most common malignancies in China. ${ }^{1}$ Moreover, it is the fifth most frequently occurring cancer and the third leading cause of cancer deaths worldwide, especially in China, Japan, South America, and Eastern Europe, which also report the highest incidence of gastric cancer., ${ }^{2,3}$ The average 5 -year survival rate for gastric cancer ranges from $50 \%$ to $70 \%$ in Japan, and only from $10 \%$ to $30 \%$ in the other countries. ${ }^{4,5}$ Thus, novel therapeutic approaches for the effective treatment of gastric cancer patients are much needed given their current prognosis.

Current methods for treating gastric cancer include the traditional surgical resection, radiotherapy, and chemotherapy. However, these methods are followed by many adverse reactions ${ }^{4,6}$ Signal transducer and activator of transcription 3 (STAT3) is constitutively activated in various human cancer cells, such as gastric cancer cells, colon cancer cells, and breast cancer cells. ${ }^{7}$ STAT3 has been found to play an important role in preventing cell apoptosis and stimulating cell proliferation in tumor progression. ${ }^{8,9}$ Specifically, STAT3 and their downstream signaling molecules are critical to the growth of gastric cancer cells. ${ }^{10}$ Therefore, intensive efforts have been spent on developing drugs that target the STAT3 pathway for the treatment of cancer. ${ }^{11,12}$ Napabucasin, a STAT3 inhibitor, has been granted orphan drug status for the treatment of gastric cancers, including gastroesophageal junction cancer. ${ }^{13-15}$ Overall, studies have identified the STAT3 pathway as a target for the effective prevention and treatment of gastric cancer, but clinically available small-molecule inhibitors of the STAT3 pathway are still lacking.

Nifuratel, an analogue of nifuroxazide (Figure 1A), has been clinically proven to be safe and effective in the treatment of bacterial vaginosis (BV), Candida, or Trichomonas 
A<smiles>CSCC1CN(N=Cc2ccc([N+](=O)[O-])o2)C(=O)O1</smiles>

B

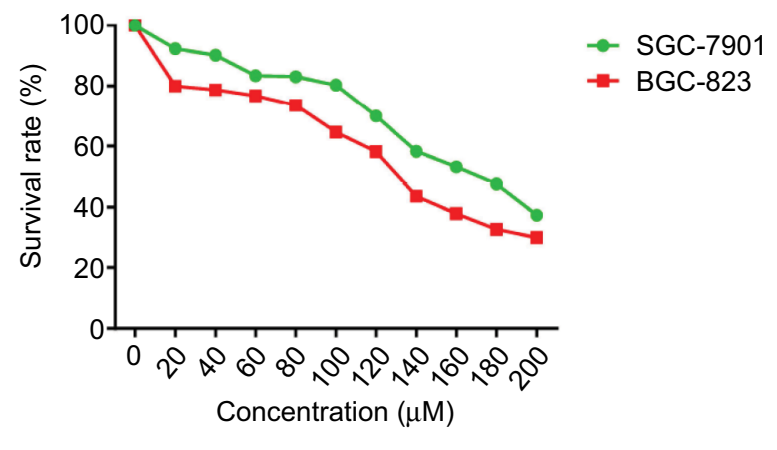

Nifuroxazide

C

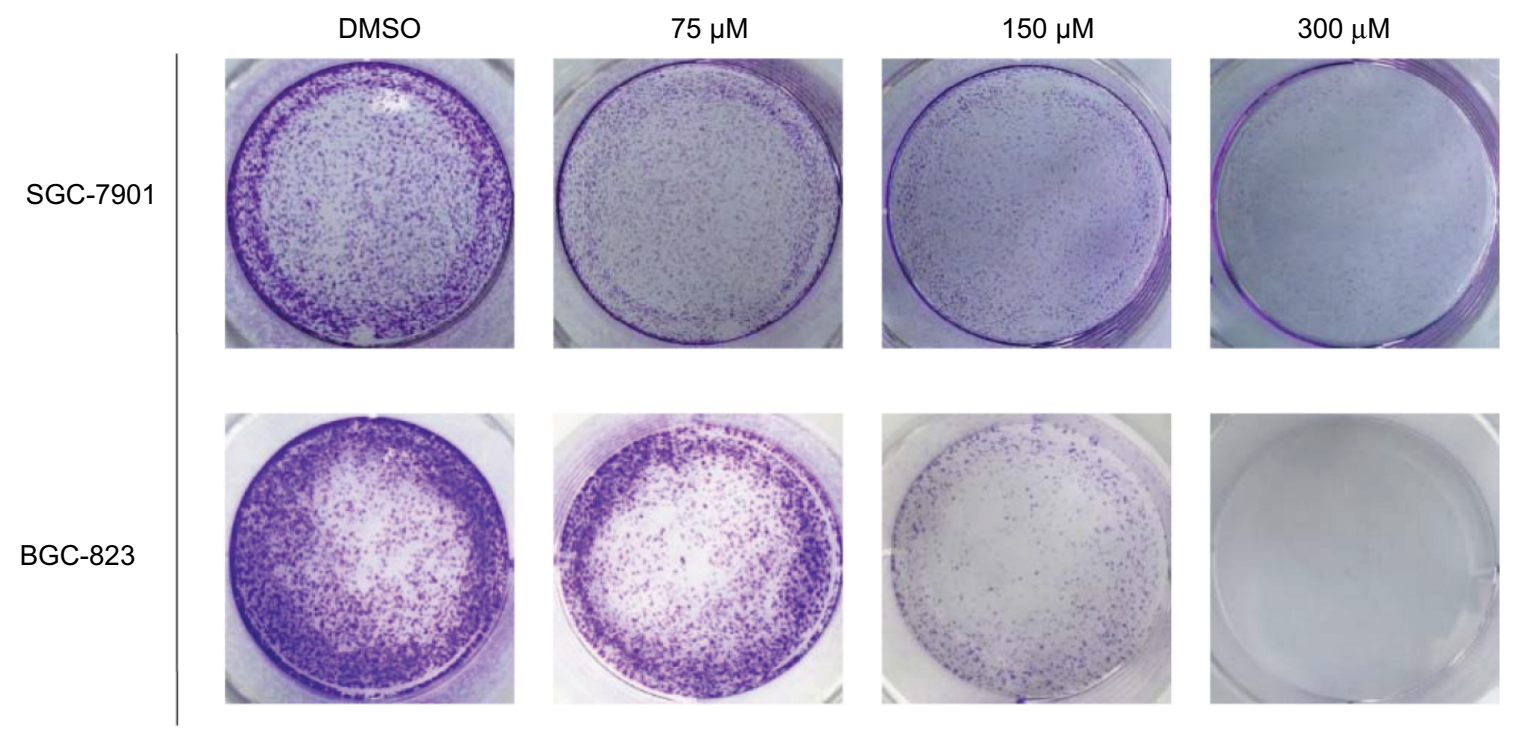

Figure I Nifuratel inhibits viability and colony formation of gastric cancer cells.

Notes: (A) Chemical structure of nifuratel and nifuroxazide. (B) Effects of nifuratel on human gastric cancer cell viability. SGC-790I and BGC-823 cell lines were treated with various concentrations of nifuratel for 48 hours and were analyzed by the MTT assay. (C) SGC-790I and BGC-823 cell lines were treated with a concentration gradient of nifuratel for 24 hours and incubated for I week, then the clones were fixed with ice-cold methanol and stained with $1 \%$ crystal violet. Abbreviation: DMSO, dimethyl sulfoxide.

vaginalis. ${ }^{16,17}$ Previous studies found have that nifuroxazide exerts potent antitumor and antimetastatic activities by inhibiting the STAT3 pathway. ${ }^{18,19}$ The aim of this study was to explore whether nifuratel exerts antitumor growth activity by inhibiting cell proliferation and inducing cell apoptosis of gastric cancer cells through downregulation of the STAT3 signaling pathway. Our results suggest that nifuratel inhibited the proliferation and induced apoptosis of gastric cancer cells. Further investigation revealed that nifuratel blocked the interleukin-6 (IL-6)-mediated activation of the STAT3 signaling pathway. This might be one of the possible mechanisms by which the expression of proapoptotic proteins was upregulated and the proliferation of gastric cancer cells was inhibited.
In the present study, the results of the MTT assay revealed that nifuratel decreased the viability of SGC-7901 and BGC-823 cells with $\mathrm{IC}_{50}$ values of 169.7 and $133.7 \mu \mathrm{M}$, respectively. Western blot analysis showed that nifuratel significantly reduced the expression of P-STAT3, without affecting the expression of P-STAT1. Additionally, nifuratelinduced apoptosis in gastric cancer cells and arrested the cell cycle in the G2/M phase. After treatment with nifuratel, the expression of Bax protein was significantly upregulated, while Bcl-2 protein was decreased in SGC-7901 and BGC-823 cells. In conclusion, our findings suggest that nifuratel can act as an anticancer agent in gastric cancer by inhibiting STAT3 signaling. 


\section{Materials and methods \\ Cell lines and antibody}

Human gastric cancer cell lines SGC-7901 and BGC-823 were obtained from the Institute of Biochemistry and Cell Biology, Chinese Academy of Sciences (Shanghai, China). Roswell Park Memorial Institute (RPMI) 1640 medium supplemented with $10 \%$ heat-inactivated fetal bovine serum (FBS) was obtained from Thermo Fisher Scientific (Waltham, MA, USA). The cells were cultured in a humidified cell incubator with an atmosphere of $5 \% \mathrm{CO}_{2}$ at $37^{\circ} \mathrm{C}$. The antibodies against P-STAT3, STAT3, P-STAT1, STAT1, Bcl-2, Bax, GAPDH, horseradish peroxidase (HRP)-conjugated donkey anti-rabbit IgG and HRP-conjugated goat anti-mouse IgG were purchased from Santa Cruz Biotechnology Inc. (Dallas, TX, USA). The Annexin V-FITC apoptosis Detection Kit I and propidium iodide (PI) were purchased from BD Pharmingen (Franklin Lakes, NJ, USA).

\section{MTT cell viability assay}

Cells were seeded into 96-well plates at a density of 8000 cells per well and cultured in RPMI 1640 medium containing $10 \%$ heat-inactivated FBS overnight. Nifuratel was dissolved in DMSO to a final concentration of 10, 20, 40, 60, 80, 100, $120,150,200$, and $300 \mu \mathrm{M}$ and gastric cancer cell lines were incubated with nifuratel for 24 hours; the $\mathrm{IC}_{50}$ values were determined by the MTT assay.

\section{Cell cycle analysis}

Cells were seed into six-well plates for 24 hours, and then treated with DMSO or different concentrations of nifuratel for 24 hours. Cells were then labeled with PI, and the cell cycle was analyzed by flow cytometry analysis (BD Biosciences, San Jose, CA, USA).

\section{Cell apoptosis analysis}

SGC-7901 and BGC-823 cells were seeded in six-well plates and grown overnight, and then treated with different concentrations of nifuratel for 24 hours. The cells were harvested and washed twice with ice-cold PBS, and stained with FITCconjugated Annexin V and PI in the binding buffer for 30 minutes. The stained cells were evaluated for apoptosis by flow cytometry analysis (FACSCalibur flow cytometer; BD Biosciences).

\section{Western blot analysis}

Cells were lysed in protein lysis buffer and centrifuged at $12,000 \mathrm{rpm}$ for 10 minutes at $4^{\circ} \mathrm{C}$ to remove nuclei and cell debris. The cell extract concentrations were determined with the Bradford protein assay kit (Bio-Rad Laboratories Inc., Hercules, CA, USA). After addition of sample loading buffer, proteins were separated by electrophoresis and the proteins were then electroblotted to poly-vinylidene difluoride transfer membranes. The blots were blocked for 2 hours with fresh 5\% non-fat milk in Tris-buffered saline with Tween 20 (TBST) buffer at room temperature, followed by incubation with primary antibody in TBST overnight at $4^{\circ} \mathrm{C}$. After washing three times with TBST, the blots were incubated with horseradish peroxidase-conjugated secondary antibodies for 1 hour. After three washes with TBST, the antibody staining was visualized using the ECL kit (Bio-Rad Laboratories Inc.). Then, the images were analyzed using the Image J computer software (National Institute of Health, Bethesda, MD, USA).

\section{Results}

The effect of nifuratel on gastric cancer cell proliferation by the MTT and colony formation assays

To investigate the effect of nifuratel on human gastric cancer cell lines, cell viability was evaluated by the MTT assay. The SGC7901 and BGC-823 cell lines were treated with increasing doses of nifuratel for 48 hours, and the cell viability was evaluated by the MTT assay. The results in Figure 1B show that cell viability was suppressed in a dose-dependent manner in the SGC-7901 and BGC-823 cell lines, with $\mathrm{IC}_{50}$ values of $169.7 \pm 2.2 \mu \mathrm{M}$ in SGC-7901 cells and $133.7 \pm 0.85 \mu \mathrm{M}$ in BGC-823cells.

Also, as shown in Figure 1C, compared with control cells, a dose-dependent decrease in colony formations by SGC7091 and BGC-823 cells was detected using a colony formation assay. These results suggest that nifuratel significantly inhibited the growth and the proliferation of human gastric cancer cells, inducing cell death in a dose-dependent manner.

\section{Nifuratel induces G2/M cell cycle arrest in human gastric cancer cells}

The cell cycle is a process involving a series of cellular events that lead to cell division and ultimately to proliferation. The entire cell cycle can be divided into four stages including the G1 phase, S phase, G2 phase, and M phase (the division stage).$^{20}$ To evaluate the cell cycle arrest associated with nifuratel, SGC-7901 and BGC-823 cell lines were treated with nifuratel for 24 hours. The percentage of nifuratel-treated and untreated gastric cancer cells in the G2/M phase was measured by flow cytometry analysis. As show in Figure 2A and $\mathrm{B}$, nifuratel significantly induced the arrest of gastric 
A

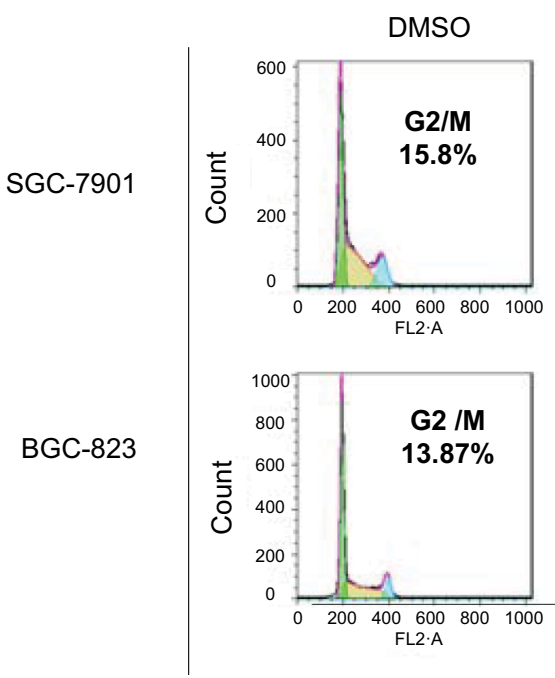

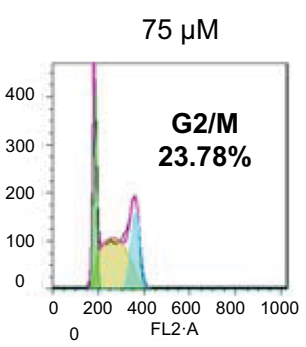
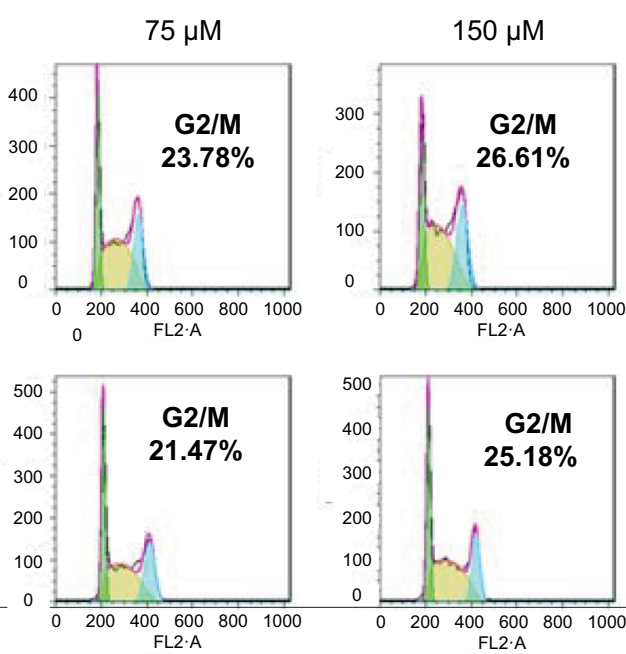

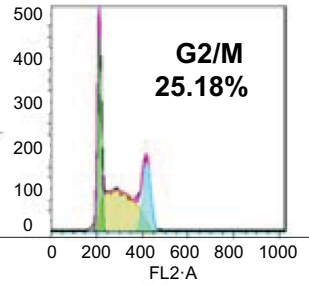

$300 \mu \mathrm{M}$
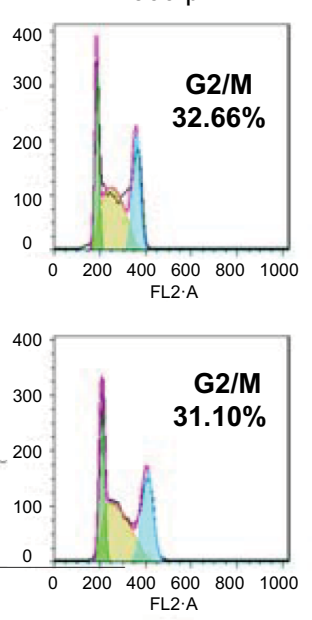

B
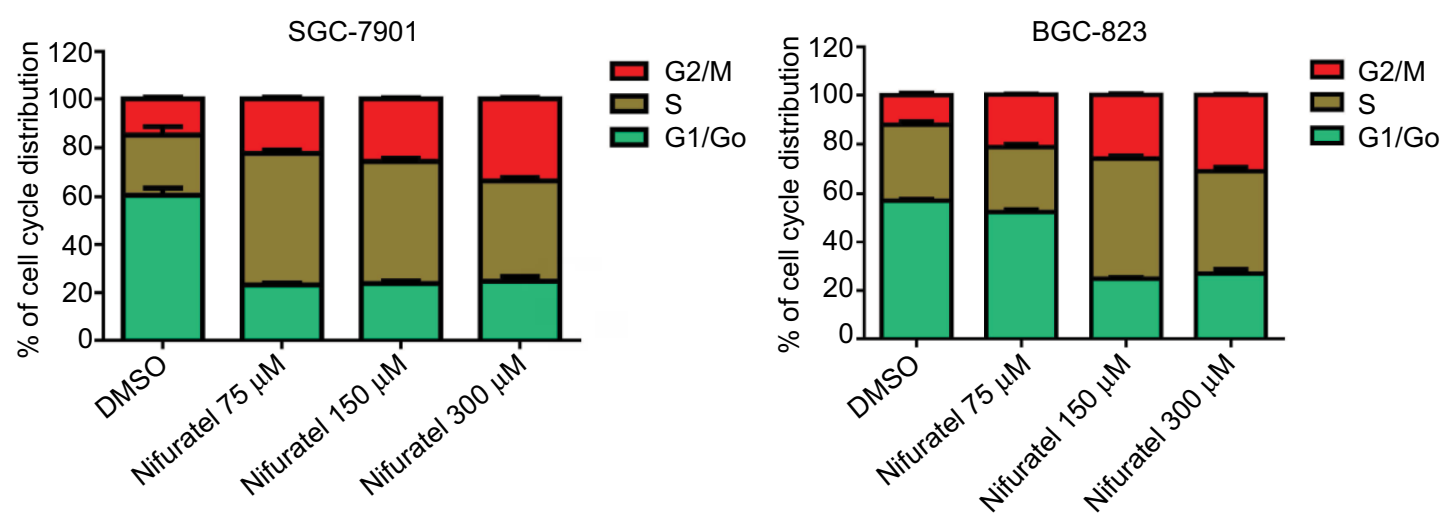

Figure 2 Nifuratel induces G2/M cell cycle arrest in human gastric cancer cell lines.

Notes: (A) Induction of cell cycle arrest in human gastric cancer cells analyzed by flow cytometry after treatment with a concentration gradient of nifuratel for 24 hours; DMSO is used as a loading control. (B) Representative histograms from flow cytometry analysis in the two human gastric cancer cells treated with various concentrations of nifuratel. Assays were performed in triplicate.

Abbreviation: DMSO, dimethyl sulfoxide.

cancer cells in the G2/M phase of the cell cycle compared with the untreated control group.

\section{Nifuratel induces apoptosis in human gastric cancer cells}

To evaluate the apoptosis-inducing effect of nifuratel in human gastric cancer cell lines, the cancer cells were treated with nifuratel for 24 hours, stained with Annexin V-FITC and PI, and apoptotic cells were evaluated by flow cytometry analysis. The data showed that nifuratel dose-dependently induced cell apoptosis (Figure 3A). In addition, Western blot analysis data further revealed that nifuratel increased the expression of the proapoptotic protein Bax and decreased the level of the antiapoptotic protein Bcl-2 in human gastric cancer cells in a dose-dependent manner (Figure 3B). Our results also revealed that nifuratel could regulate the expression of cell death-related proteins, which blocks the process of human gastric cancer cell proliferation.

\section{Nifuratel inhibits the STAT3 signaling pathway}

A number of studies have shown that overexpression of the STAT3 protein may be associated with the progression of gastric cancer. ${ }^{21}$ Our results indicated that treatment with $300 \mu \mathrm{M}$ nifuratel markedly reduces the phosphorylated STAT3 (P-STAT3) protein level in SGC-7901 and BGC-823 cell lines. As show in Figure 4, nifuratel decreased P-STAT3 protein expression in SGC-7901 and BGC-823 cell lines in 
A

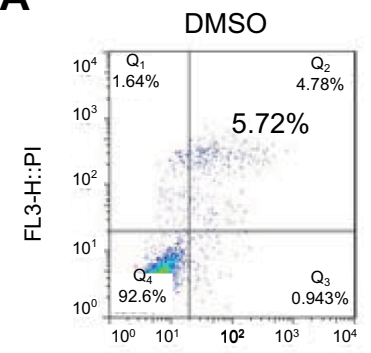

FLA-H::AnnexinV-FITC

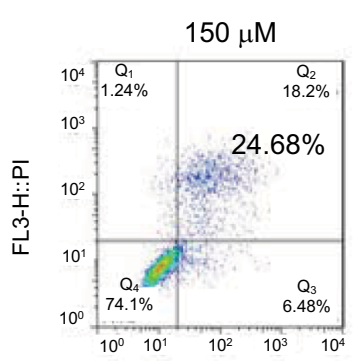

FLA-H::AnnexinV-FITC

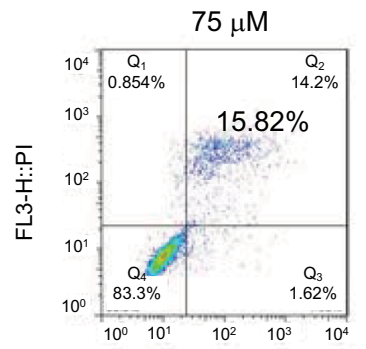

FLA-H::AnnexinV-FITC

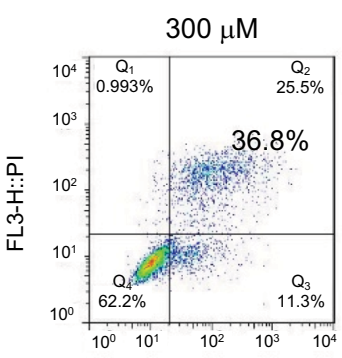

FLA-H::AnnexinV-FITC

SGC-7901

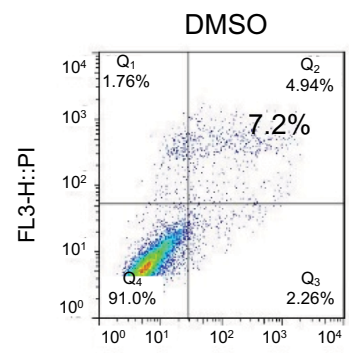

FLA-H::AnnexinV-FITC

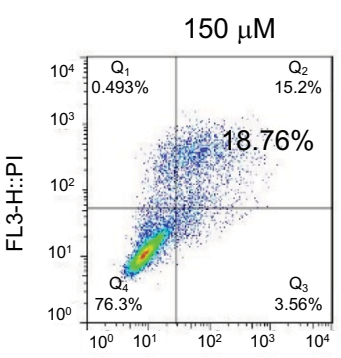

FLA-H::AnnexinV-FITC

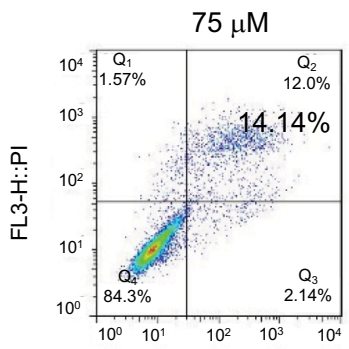

FLA-H::AnnexinV-FITC

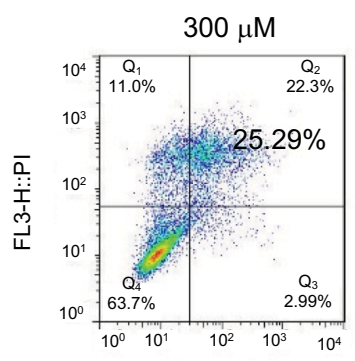

FLA-H::AnnexinV-FITC

BGC-823

B

SGC-7901

BGC-823
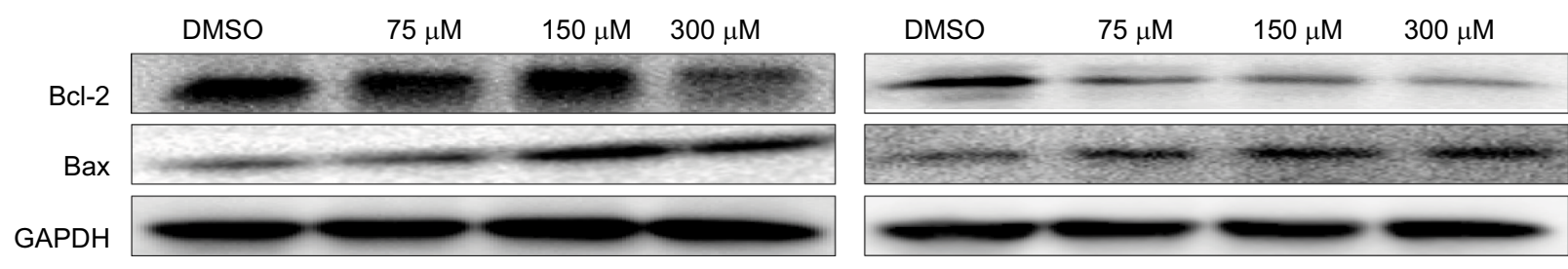

Figure 3 Nifuratel-induced apoptosis of gastric cancer cells.

Notes: (A) SGC-790I and BGC-823 cell lines were treated with a concentration gradient of nifuratel for 24 hours and stained with Annexin V and propidium iodide. The results were assessed by flow cytometry analysis. (B) Western blot analysis of apoptosis-related proteins. The expression of Bcl-2 and Baxin in SGC-790I and BGC-823 cells were measured by Western blotting after incubation with nifuratel for 24 hours.

Abbreviation: DMSO, dimethyl sulfoxide.

A

SGC-7901

DMSO $\quad 75 \mu \mathrm{M} \quad 150 \mu \mathrm{M} \quad 300 \mu \mathrm{M}$

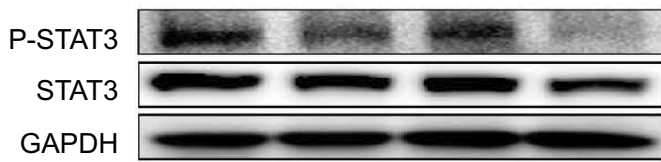

SGC-7901

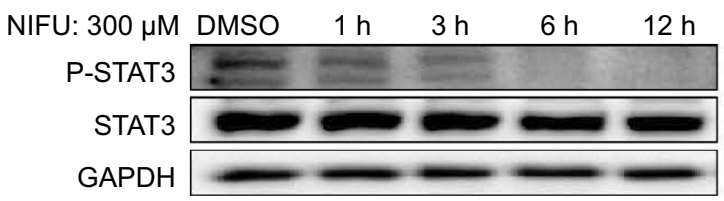

BGC-823
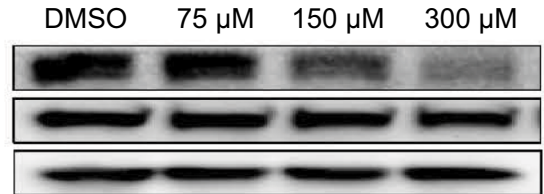

BGC-823

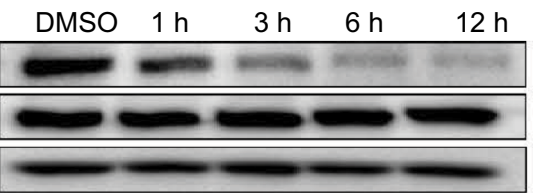

Figure 4 Western blot analysis of total STAT3 and P-STAT3 in SGC-790I and BGC-823 cells.

Notes: (A) SGC-790I and BGC-823 cell lines were treated with a concentration gradient of nifuratel, the expression of P-STAT3 is shown by Western blot analysis. (B) The expression of P-STAT3 time dependently induced by nifuratel is shown by Western blot analysis in SGC-790I and BGC-823 cell lines.

Abbreviations: DMSO, dimethyl sulfoxide; NIFU, nifuratel; STAT3, signal transducer and activator of transcription 3; P-STAT3, phosphorylated STAT3. 
A

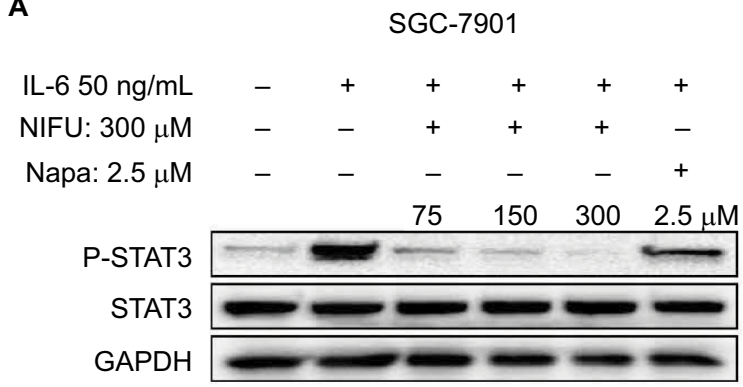

B

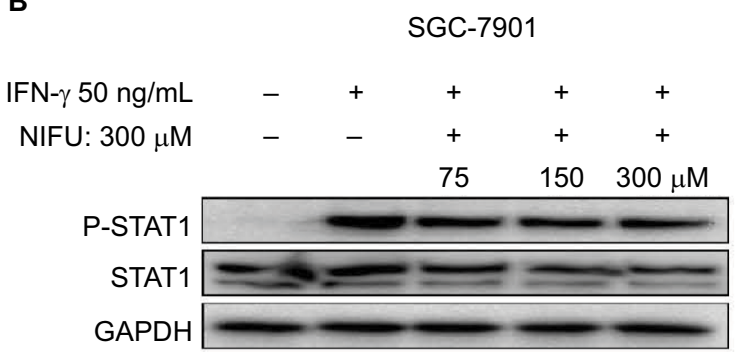

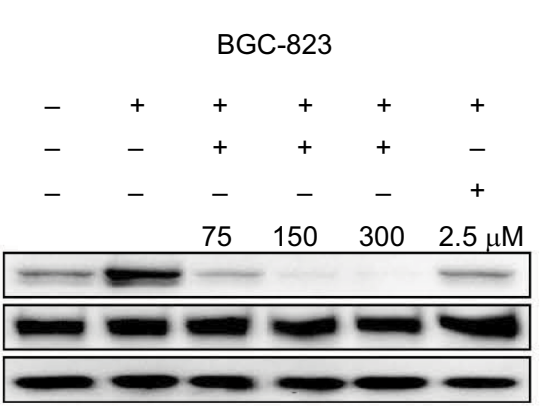

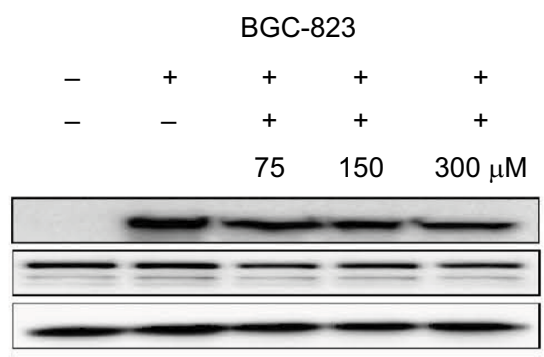

Figure 5 Nifuratel blocked IL-6-induced STAT3 phosphorylation.

Notes: (A) Gastric cancer cells were pretreated with the indicated concentrations of nifuratel and napabucasin for 24 hours and then stimulated with IL-6 ( 50 ng/ $\mathrm{L}$ ) for 30 minutes. Whole-cell extracts were prepared and subjected to Western blot analysis using the indicated antibodies. (B) Gastric cancer cells were pretreated with the indicated concentrations of nifuratel for 24 hours and then stimulated with interferon- $\gamma($ IFN- $\gamma)(50 \mathrm{ng} / \mu \mathrm{L})$ for 30 minutes. Whole-cell extracts were prepared and subjected to Western blot analysis using the indicated antibodies.

Abbreviations: NIFU, nifuratel; Napa, napabucasin; P-STAT3, phosphorylated STAT3; STAT3, signal transducer and activator of transcription 3.

a concentration-dependent manner after 24-hour treatment. Notably, the $300 \mu \mathrm{M}$ dose of nifuratel also markedly decreased P-STAT3 protein expression after 6 hours in the SGC-7901 cell lines and after 3 hours in the BGC-823 cell lines.

Importantly, the previous study found that the IL-6-induced phosphorylation of STAT3 in neoplastic gastric tissue positively correlated with tumor progression. ${ }^{22}$ It has been established that IL-6-mediated activation of STAT3 signaling pathway can promote the progression of other cancers. ${ }^{23,24}$ The STAT3 inhibitor inhibited the activation of STAT3, blocking the IL-6 signaling pathway which downregulated cancer cell proliferation, and downregulated the antiapoptotic protein $\mathrm{Bcl}-2 .{ }^{25}$ Thus, to further clarify whether nifuratel can block the IL-6-induced phosphorylation of STAT3, we combined the treatment with nifuratel and IL-6. In addition, napabucasin, an orally available STAT3 inhibitor, was used as a positive control group. The results indicated that nifuratel could block the IL-6-induced activation of the STAT3 signaling pathway, but had no effect on the activation of STAT1 mediated by interferon- $\gamma$ (IFN- $\gamma$ ) (Figure 5). Our results also revealed that nifuratel regulated the expression of the cell death-related protein Bcl-2, induced cell cycle arrest, and inhibited the proliferation of human gastric cancer cells by blocking the IL-6-induced activation of the STAT3 signaling pathway.

\section{Discussion}

A tumor develops from cell differentiation abnormalities, excessive proliferation, inhibition of apoptosis, and other factors. ${ }^{2}$ The abnormalities of signal transduction pathways play an important role in these processes. ${ }^{26,27}$ Several studies have reported that STAT3 is continuously activated in most human primary cancer foci and tumor cell lines, such as human astrocytoma, multiple osteosarcoma, prostate cancer, colon cancer, gastric cancer, etc. ${ }^{8,10,28}$

Our study showed that nifuratel can reduce the viability of gastric cancer cells and stimulate apoptosis in human gastric cancer cells in a dose-dependent manner. In addition, nifuratel induced the arrest of gastric cancer cells in the G2/M phase of the cell cycle. Western blot analysis results suggest that the antitumor activity of nifuratel might be mediated via the STAT3 inhibition. Furthermore, nifuratel can block the IL-6-induced activation of the STAT3 signaling pathway, thereby inhibiting the proliferation of tumor cells. Our study further confirmed that the treatment with nifuratel can upregulate the expression of the proapoptotic protein Bax and downregulate the expression of the antiapoptotic protein Bcl-2. We have demonstrated that nifuratel inhibited the IL-6-induced activation of the STAT3 signaling pathway and thereby induced apoptosis and reduced the growth of human gastric cancer cells. 
Nifuratel is used to effectively and safely treat bacterial vaginitis, Chlamydia trachomatis, and Mycoplasma spp., as well as fungal infections by Candida spp., for several decades. ${ }^{16}$ Additionally, though to a lesser extent, it has been reported to possess anticancer properties. Our study confirms that the clinical drug nifuratel possesses anticancer properties against tumor cells. We explored the novel antitumor effects of nifuratel and tried to repurpose it for treating gastric cancer. Additionally, we plan to enhance the selectivity and reduce the toxicity effects of nifuratel on normal cells. We will also investigate the combination of nifuratel and other clinical cancer drugs to improve the anticancer potency of nifuratel and verify its effect on human gastric cancer in vivo.

\section{Acknowledgments}

This research was supported by the Zhejiang Provincial Natural Science Fund (LY16H160052), Medical Scientific Research Fund of Zhejiang Province (2017192276), and Wenzhou Science and Technology Project (Y20160052). We also thank the Chinese Society of Clinical Oncology (CSCO Y-MX2016-058) and Zhejiang Anticancer Association.

\section{Disclosure}

The authors report no conflicts of interest in this work.

\section{References}

1. Chen W, Zheng R, Baade PD, et al. Cancer statistics in China, 2015. CA Cancer J Clin. 2016;66(2):115-132.

2. Siegel RL, Miller KD, Jemal A. Cancer Statistics, 2017. CA Cancer J Clin. 2017;67(1):7-30.

3. Siegel RL, Miller KD, Jemal A. Cancer statistics, 2016. CA Cancer J Clin. 2016;66(1):7-30.

4. Shitara K, Kadowaki S, Nishina T, et al. Safety, pharmacokinetic, and clinical activity profiles of ramucirumab in combination with three platinum/fluoropyrimidine doublets in Japanese patients with chemotherapy-naive metastatic gastric/gastroesophageal junction cancer. Gastric Cancer. Epub 2017 Jun 30.

5. Kodera Y, Fujitani K, Fukushima N, et al. Surgical resection of hepatic metastasis from gastric cancer: a review and new recommendation in the Japanese gastric cancer treatment guidelines. Gastric Cancer. 2014;17(2):206-212.

6. Thuss-Patience PC, Shah MA, Ohtsu A, et al. Trastuzumab emtansine versus taxane use for previously treated HER2-positive locally advanced or metastatic gastric or gastro-oesophageal junction adenocarcinoma (GATSBY): an international randomised, open-label, adaptive, phase 2/3 study. Lancet Oncol. 2017;18(5):640-653.

7. Yu H, Pardoll D, Jove R. STATs in cancer inflammation and immunity: a leading role for STAT3. Nat Rev Cancer. 2009;9(11):798-809.

8. Yu H, Lee H, Herrmann A, Buettner R, Jove R. Revisiting STAT3 signalling in cancer: new and unexpected biological functions. Nat Rev Cancer. 2014;14(11):736-746.
9. Zhao C, Li H, Lin HJ, Yang S, Lin J, Liang G. Feedback activation of STAT3 as a cancer drug-resistance mechanism. Trends Pharmacol Sci. 2016;37(1):47-61.

10. Huynh J, Etemadi N, Hollande F, Ernst M, Buchert M. The JAK/STAT3 axis: a comprehensive drug target for solid malignancies. Semin Cancer Biol. 2017;45:13-22.

11. Lin L, Hutzen B, Zuo M, et al. Novel STAT3 phosphorylation inhibitors exhibit potent growth-suppressive activity in pancreatic and breast cancer cells. Cancer Res. 2010;70(6):2445-2454.

12. Rath KS, Naidu SK, Lata P, et al. HO-3867, a safe STAT3 inhibitor, is selectively cytotoxic to ovarian cancer. Cancer Res. 2014;74(8): 2316-2327.

13. Hubbard JM, Grothey A. Napabucasin: an update on the first-in-class cancer stemness inhibitor. Drugs. 2017;77(10):1091-1103.

14. Jonker DJ, Nott L, Yoshino T, et al. A randomized phase III study of napabucasin [BBI608] (NAPA) vs placebo (PBO) in patients (pts) with pretreated advanced colorectal cancer (ACRC): the CCTG/AGITG CO.23 trial. Ann Oncol. 2016;27(6):149-206.

15. Grothey A, Tebbutt N, Van Cutsem E, et al. CanStem303C trial: a phase III study of BBI-608 (napabucasin) in combination with 5-fluorouracil (5-FU), leucovorin, irinotecan (FOLFIRI) in adult patients with previously treated metastatic colorectal cancer (mCRC). Ann Oncol. 2016;27(6):149-206.

16. Polatti F. Bacterial vaginosis, Atopobium vaginae and nifuratel. Curr Clin Pharmacol. 2012;7(1):36-40.

17. Brumfitt W, Reynolds AV, Hamilton-Miller JM. Letter: activity of nitrofurantoin and nifuratel against anaerobic Gram-negative bacilli. Lancet. 1975;1(7904):460.

18. Ye TH, Yang FF, Zhu YX, et al. Inhibition of Stat3 signaling pathway by nifuroxazide improves antitumor immunity and impairs colorectal carcinoma metastasis. Cell Death Dis. 2017;8(1):e2534.

19. Nelson EA, Walker SR, Kepich A, et al. Nifuroxazide inhibits survival of multiple myeloma cells by directly inhibiting STAT3. Blood. 2008;112(13):5095-5102.

20. Jang W, Kim T, Koo JS, Kim SK, Lim DS. Mechanical cue-induced YAP instructs Skp2-dependent cell cycle exit and oncogenic signaling. EMBO J. 2017;36(17):2510-2528.

21. Wang TT, Zhao YL, Peng LS, et al. Tumour-activated neutrophils in gastric cancer foster immune suppression and disease progression through GM-CSF-PD-L1 pathway. Gut. Epub 2017 Mar 8.

22. Howlett M, Menheniott TR, Judd LM, Giraud AS. Cytokine signalling via gp130 in gastric cancer. Biochim Biophys Acta. 2009;1793(11): $1623-1633$.

23. Long KB, Tooker G, Tooker E, et al. IL-6 receptor blockade enhances chemotherapy efficacy in pancreatic ductal adenocarcinoma. Mol Cancer Ther. 2017;16(9):1898-1908.

24. Bharti R, Dey G, Banerjee I, et al. Somatostatin receptor targeted liposomes with Diacerein inhibit IL-6 for breast cancer therapy. Cancer Lett. 2017;388:292-302.

25. Bharti R, Dey G, Ojha PK, et al. Diacerein-mediated inhibition of IL-6/ IL-6R signaling induces apoptotic effects on breast cancer. Oncogene. 2016;35(30):3965-3975.

26. Medlock J, Das AAK, Madden LA, Allsup DJ, Paunov VN. Cancer bioimprinting and cell shape recognition for diagnosis and targeted treatment. Chem Soc Rev. 2017;46(16):5110-5127.

27. Miller MA, Weissleder R. Imaging of anticancer drug action in single cells. Nat Rev Cancer. 2017;17(7):399-414.

28. Jung KH, Yoo W, Stevenson HL, et al. Multi-functional effects of a small-molecule STAT3 inhibitor on NASH and HCC in mice. Clin Cancer Res. 2017;23(18):5537-5546. 


\section{Publish your work in this journal}

Cancer Management and Research is an international, peer-reviewed open access journal focusing on cancer research and the optimal use of preventative and integrated treatment interventions to achieve improved outcomes, enhanced survival and quality of life for the cancer patient. The manuscript management system is completely online and includes a very quick and fair peer-review system, which is all easy to use. Visit http://www.dovepress.com/testimonials.php to read real quotes from published authors.

Submit your manuscript here: https://www.dovepress.com/cancer-management-and-research-journal 